\begin{tabular}{lcc}
$\begin{array}{l}\text { Abstract IDDF2018-ABS-0135 Table } 2 \\
\text { regression for risk factors of ACN }\end{array}$ & Multivariate binary \\
\hline $\begin{array}{l}\text { Obesity indexes } \\
\text { Adjusted odds ratio(95\% Cl) with age, gender, family } \\
\text { history CRC in FDR, diabetes }\end{array}$ & \begin{tabular}{l} 
p-value \\
\hline$B M I \geq 23$
\end{tabular} \\
$B M I \geq 25$ & $1.60(1.08,2.36)$ & 0.018 \\
$W C($ male $90 \mathrm{~cm}$, & $1.44(1.01,2.10)$ & 0.044 \\
female $85 \mathrm{~cm})$ & $1.33(0.91,1.95)$ & 0.140 \\
$W C($ male $85 \mathrm{~cm}$, & $1.52(1.03,2.24)$ & 0.035 \\
female $80 \mathrm{~cm})$ & & \\
\hline
\end{tabular}

multivariate regression results to select the one with a highest odds ratio $(\mathrm{OR})$ as the best predictor. The risk scores of all predictors and C-statistics was reported for the regression model with obesity index having the highest OR.

Results A total of 2813 subjects were enrolled (mean age $58.7 \pm 8.4$ years, $52.0 \%$ male), of which $142(5.0 \%)$ was diagnosed with ACN. In univariate regression, several $\mathrm{BMI} / \mathrm{WC}$ criteria were significant, with age, gender, family history, smoking status and diabetes (table 1). The Asian criteria for overweight (BMI $\geq 23$ ) attained the highest adjusted OR (1.60, 95\% confidence interval (CI) $1.08,2.36)$, when compared with international criteria for overweight (BMI $\geq 25$ ) (OR 1.46, 95\% CI 1.01, 2.10) and Asian criteria for abdominal obesity (male $\geq 85 \mathrm{~cm}$, female $\geq 80 \mathrm{~cm}$ ) (OR 1.52, 95\% CI 1.03, 2.24) (table 2). The C-statistics of the new model with BMI $\geq 23$ as the predictor for ACN was 0.648 (95\% CI 0.600, 0.696).

Conclusions The Asian criteria for obesity had the most discriminatory performance to predict $\mathrm{ACN}$ as compared to other obesity measures in this asymptomatic Chinese cohort. These findings imply $\mathrm{BMI} \geq 23$ may be most suitable 'obesitypredictor' of ACN in risk algorithms.

\section{IDDF2018-ABS-0136 A SCORING SYSTEM FOR OUTPATIENT IMPROVEMENT IN THE DIAGNOSTIC YIELD OF UPPER GASTROINTESTINAL ENDOSCOPY}

Nitin Agarwal*. Postgraduate Institute of Medical Education and Research, Dr. Ram Manohar Lohia Hospital, Delhi, India

\subsection{6/gutjnl-2018-IDDFabstracts. 123}

Background With the widespread availability of diagnostic procedures, it has become necessary to evaluate the efficacy and appropriateness, especially with regard to gastrointestinal endoscopy (upper and lower). Many centres have adopted an open-access referral policy, resulting in increased costs, waiting times and clinical workload. Attempts have been made to rationalise resources, by using limited indications. Diagnostic accuracy for dyspepsia is only $40 \%-50 \%$. We planned to develop a scoring system which would help in differentiating disease from non-diseased and improve the diagnostic yield of upper gastrointestinal endoscopy.

Methods A total of 150 patients from the outpatient and inpatient departments of a tertiary care hospital in north India over 2 years were included. The clinical history and examination of the patients were recorded on a performa. The examination was undertaken by a standard forward-viewing fibre optic endoscope using topical anaesthesia by the consultant. The outcome of the endoscopy was categorised as positive if there was a significant finding. Less severe and equivocal findings such as gastritis, duodenitis were not considered positive for this study. Logistic regression (forward LR score) was used; the coefficient of regression was used to assign a score for each symptom.

Results Pain was the most common symptom; in 110 patients (77.3\%) the endoscopy showed no significant findings. Significant findings were seen in 34 patients; diagnostic yield $=22.7 \%$. Clinical features like weight loss, hemaemesis, malena, dysphagia, anaemia, abdominal distention, ascites and abdominal lump significantly discriminated and pointed towards a positive endoscopic finding. The pain was not a good discriminating factor; dysphagia, the presence of ascites and the presence of abdominal lump independently predict significant endoscopic findings. At a total score of 4 or less we can avoid doing endoscopy in 75 (50\%); as the score increase the sensitivity decreases while the specificity increases. At a score of 5 the sensitivity $=82 \%$ and specificity $=71 \%$. All 11 malignancies had a score $=5$ or more.

Conclusions Our findings suggest how the selection of patients for endoscopy can be improved. It also provides a basis for prospective studies which can lead to better use of resources in future.

\section{IDDF2018-ABS-0141 AN ANALYSIS OF THE EFFICACY AND SAFETY OF INFLIXIMAB IN THE MANAGEMENT OF REFRACTORY ULCERATIVE COLITIS AND ULCERATIVE COLITIS WITH EXTRA-INTESTINAL MANIFESTATIONS}

Hui Su*, Yan Jia, Yuanming Pan, Xiaojuan Lu, Ruying Fan, Xin Fan, Yilin Wang, Shirong Li, Jianqiu Sheng. Department of Gastroenterology, PLA Army General Hospital, Beijing, China

\subsection{6/gutjnl-2018-IDDFabstracts. 124}

Background To evaluate the efficacy and safety of Infliximab (IFX) in the management of refractory ulcerative colitis (UC) and UC with extra-intestinal manifestations.

Methods The clinical data for 36 cases including refractory UC patients and UC patients with special extra-intestinal manifestations, who all accepted treatment with IFX, were analysed retrospectively.

Results Among all the 36 cases, 33 were refractory UC (5 with extra-intestinal manifestations) and another 3 with special extra-intestinal manifestations ( 2 with perianal abscess and 1 with ankylosing spondylitis). After treatment with IFX, 13 patients achieved clinical remission, of whom 3 achieved mucosal healing, 15 achieved clinical response and 8 failed to respond. The total effective rate of IFX treatment was $77.78 \%$, remission rate $36.11 \%$; Among 8 cases with extraintestinal manifestations, 5 patients' extra-intestinal manifestations improved and the efficacy rate was $62.5 \%$. No serious adverse events occurred during observation.

Conclusions IFX is effective and safe in the management of refractory UC and extra-intestinal manifestations. 